\title{
Creación de Videojuegos como Estrategia Educativa en Algoritmia
}

\section{Creation of Video Games as an Educational Strategy in Algorithms}

Lizzie Edmea Narváez-Díaz y Rocio López-Martínez ${ }^{2}$

\section{EnICIÓN. Resiliencia}

Recibido: 9/abril/2021

Aceptado: $13 /$ julio/2021

Publicado: 29/noviembre/2021

$\downarrow$ Páginas: 22-30

\&aís

${ }^{1}$ México

${ }^{2}$ México

\section{IIIII Institución}

${ }^{1}$ Universidad Autónoma de Yucatán

${ }^{2}$ Universidad Autónoma de Querétaro

\section{Correo Eletrónico}

1'lendiaz@correo.uady.mx

2rocio.edith.lopez@uaq.mx

\section{ORCID}

${ }^{1}$ https://orcid.org/0000-0003-0595-1932 ${ }^{2} \mathrm{https}: / /$ orcid.org/0000-0002-5209-3523

\section{Citar así: CfAPA / IEEE}

Narváez-Díaz, L. \& López-Martínez, R. (2021). Creación de Videojuegos como Estrategia Educativa en Algoritmia. Revista Tecnológica-Educativa Docentes 2.0, 1(1), 22-30.

https://doi.org/10.37843/rted.v1i1.219

L. Narváez-Díaz y R. López-Martínez, "Creación de Videojuegos como Estrategia Educativa en Algoritmia", RTED, vol. 1, n. ${ }^{\circ}$ 1, pp. 22-30, nov. 2021.

\section{Resumen}

La programación de computadoras y en especial los principios básicos de esta área son un aspecto importante. Por ello, la Universidad Autónoma de Yucatán (UADY) crea en la Unidad Multidisciplinaria Tizimín (UMT) la Licenciatura en Ingeniería de Software (LIS) y en ella se emprende a impartir la materia de Algoritmia en el primer semestre de la carrera. La introducción de alumnos al estudio de conceptos básicos de programación no es una tarea fácil, requiere tiempo, esfuerzo y un enfoque especial en la forma en que se aprende y se enseña. La presente investigación basada en lenguaje de programación Scratch fue incluido en la materia de Algoritmia con el objetivo de servir de apoyo en el entendimiento de las estructuras básicas que en ella se estudian y de este modo el alumno organizado en grupos de trabajo pudiera crear un videojuego para plasmar sus conocimientos. La inclusión de Scratch a Algoritmia, de la mano con la metodología del aprendizaje basada en proyectos y la teoría del construccionismo, permitió el desarrollo de habilidades en los alumnos para el desarrollo de sus videojuegos. El enfoque metodológico de la investigación fue mixto y la muestra estuvo formada por todos los alumnos inscritos a Algoritmia en tres períodos semestrales. Durante este tiempo se generaron 24 videojuegos los cuales cumplieron con los objetivos planteados y los resultados obtenidos de todo el proceso investigativo han sido satisfactorios.

Palabras clave: Videojuegos, scratch, algoritmia.

\section{Abstract}

Introducing students to the study of programming basics is not an easy task; it requires time, effort, and a particular focus on how it is learned and taught. Computer programming and especially the basic principles of this area are essential aspects. For this reason, the Autonomous University of Yucatán (UADY) created in the Tizimín Multidisciplinary Unit (UMT) the bachelor's degree in Software Engineering (LIS), and it began to teach the subject of Algorithmic in the first semester of the career. Included research based on the Scratch programming language was in the Algorithmic issue to support understanding the basic structures studied in it. In this way, the student-organized in workgroups could create a video game to translate their knowledge. The inclusion of Scratch to Algorithm, hand in hand with the project-based learning methodology and the theory of constructionism, allowed the students to develop their video games. The mixed methodological approach was the research approach, and the sample consisted of all the students enrolled in Algorithmic in three semester periods. During this time, 24 video games were generated which met the objectives set, and the results obtained from the entire investigative process have been satisfactory.

Keywords: Videogames, scratch, algorithms. 


\section{Introducción}

La programación de computadoras y en especial los principios básicos de esta área son un aspecto importante cuando se empieza a involucrar alumnos a la ingeniería de software, por lo que es necesario que los educandos entiendan bien los conceptos estudiados al inicio dado que los emplearán posteriormente. El curso Algoritmia que se imparte en la Licenciatura en Ingeniería de Software (LIS), en la Unidad Multidisciplinaria Tizimín (UMT), de la Universidad Autónoma de Yucatán (UADY), tiene estas características, en otras palabras, es de carácter introductorio al área y los alumnos que lo cursan no tienen un rendimiento académico adecuado, esta situación se afirma en función de los resultados obtenidos en el estudio previo realizado en Narváez \& López (2019), los cuales preocuparon a los docentes.

Narváez \& López (2019) mencionan que desde la primera vez que se impartió el curso de Algoritmia en el 2016 solo el 58.33\% de los alumnos tuvo un nivel de desempeño entre satisfactorio y sobresaliente y en el 2017 el $46.15 \%$ se encontró en ese mismo nivel, los datos arrojan un resultado no adecuado en este curso, son un indicador de que los alumnos no están adquiriendo bien los conocimientos requeridos; cabe mencionar que las dos veces referidas en que se impartió Algoritmia fue llevada a cabo del modo tradicional, sin el uso de alguna herramienta tecnológica. Es importante citar que la competencia general que se promueve en la materia es el desarrollo de algoritmos para solucionar problemas computacionales, en forma secuencial y lógica (Gómez et al., 2016).

Buscando estrategias para apoyar la situación de los alumnos, se encontraron investigaciones de interés. En Muñoz et al. (2015) citan que actividades didácticas donde se desarrollan productos como los videojuegos, aumentan la motivación para aprender y aplicar conocimientos de programación de modo tangible. Asimismo Compañ-Rosiqueet et al. (2015) describen que cuando el alumno diseña un algoritmo, le está enseñando a otro compañero cómo resolver una tarea, lo cual implica comprender lo que intenta explicar, no podrá transmitir conocimientos si no los domina. Vargas (2015) señala que la creación de videojuegos se asocia al Game-Based Learning el cual ha trascendido con diversas iniciativas que señalan al proceso de crear videojuegos como una estrategia para desarrollar habilidades como el pensamiento crítico, la resolución de problemas y el diseño creativo.

En este orden de ideas, en González et al. (2007) mencionan que existen numerosos estudios relacionados con los beneficios de los videojuegos, tal es el caso del incremento de la comprensión lectora, la promoción de la creatividad (aportando motivación), además de ser un estímulo el cual facilita el proceso de aprendizaje y aumenta la concentración así como la atención del alumno. De acuerdo con Saavedra (2020) así como con LópezEscribano \& Sánchez-Montoya (2012) al crear videojuegos el alumno se vuelve más autónomo y activo al trabajar proyectos de su interés. También es importante mencionar que los videojuegos promueven el llamado pensamiento complejo, definido como la capacidad para interconectar desde la reflexión diferentes dimensiones y disciplinas las cuales explican la realidad (AulaPlaneta, 2021).

Otro concepto que cobra relevancia en esta investigación es la programación, partiendo de este se encontró en Sleeman (1986, citado en Alania et al., 2012), que el primer objetivo de un programador es descomponer el problema para crear un plan detallado (un algoritmo), el segundo es implementar el plan en un lenguaje y el tercero es depurar el programa final. En Algoritmia se cumple el primer objetivo, se trata de construir el algoritmo sin implementarlo o depurarlo, dada la situación, el alumno no cuenta con un referente concreto para ver la ejecución de su trabajo, su solución queda plasmada en papel y lápiz lo cual no les representa mucho. Partiendo de este punto se plantea que en Algoritmia el alumno pueda implementar el algoritmo en un lenguaje y lo depure, mediante una herramienta simple e intuitiva, en este sentido se optó por usar el lenguaje de programación Scratch. 
En la investigación sobre Scratch, López (2011) señala que es un entorno de programación creado por investigadores del Instituto Tecnológico de Massachusetts, bajo la dirección del Dr. Resnick, Scratch hace la programación divertida para los que se enfrentan por primera vez a programar; se diseñó como medio de expresión para ayudar a niños y jóvenes a plasmar sus ideas creativamente, desarrollar habilidades de pensamiento lógico y de aprendizaje. Como se mencionó en esta investigación se usó Scratch porque es un lenguaje para crear programas sin poseer conocimientos profundos del código, al ser un entorno visual de programación por bloques. El diseño y la creación de proyectos en Scratch sirve de apoyo a los estudiantes para que aprendan a pensar de forma creativa, razonar sistemáticamente y a trabajar en modo colaborativo (MIT Scratch Team, 2018; Monjelat et al., 2018).

Partiendo del modelo de la UADY (2012) y de lo encontrado en investigaciones relacionadas surge una motivación en los docentes de Algoritmia para introducir cambios en ella a fin de que desde esta materia introductoria el alumno empiece a programar en forma simple, sin implicar la memorización de complicadas sintaxis o de obligarlo a aprender un lenguaje de programación a la par de su carga en el curso. Esta fase de programación a incluir por medio de la incorporación de Scratch se contempló como una estrategia de aprendizaje de apoyo en la formación de los estudiantes, para que por medio del desarrollo de videojuegos básicos pudieran entender mejor los conceptos introductorios de programación.

Este proceso investigativo se centró en el aprendizaje basado en proyectos así como también hizo uso de la teoría del construccionismo de Papert la cual se centra en el arte de aprender o aprender a aprender y se interesa en cómo los estudiantes se involucran en una conversación (propia o ajena) con artefactos tangibles (en este caso los videojuegos) y cómo estas conversaciones fomentan el aprendizaje autodirigido para facilitar la construcción de nuevos conocimientos (Ackermann, 2001, citado en Aparicio \& Ostos, 2018).

De todo este planteamiento surgió el objetivo de la investigación que fue mejorar el entendimiento de las estructuras básicas estudiadas en Algoritmia por medio de la creación de videojuegos que empleen esos conceptos, logrando un tarea colaborativa mediante equipos de trabajo; todo esto mediante la integración del lenguaje de programación Scratch a la asignatura, para que el alumno al final del curso entregue un proyecto de desarrollo de software enfocado a un videojuego básico diseñado en un lenguaje simple e intuitivo.

\section{Metodología}

El enfoque metodológico empleado fue mixto ya que se recolectaron y analizaron datos cuantitativos y cualitativos, su sustento se centra en tratar de obtener de ambos métodos la fortaleza necesaria para enriquecer la explicación y comprensión del fenómeno que se estudia, aprovechando lo mejor de ambos tipos, en la parte cuantitativa un método riguroso y sin lugar a ambigüedades, mientras que la parte cualitativa da pauta a la flexibilidad, donde el fenómeno es visto desde dentro (Guerrero-Castaneda et al., 2016). Con todo lo recabado se buscó responder el objetivo de este trabajo para avanzar hacia la comprensión de la situación analizada.

El contexto de esta investigación se encuentra delimitado por un entorno constituido por todos los alumnos de la Licenciatura en Ingeniería de Software que fueron inscritos al curso de Algoritmia, el cual se imparte en el primer semestre como una asignatura introductoria a los principios de programación de computadoras. Los alumnos participantes se corresponden con los períodos semestrales siguientes:

- Semestre agosto - diciembre 2018.

- Semestre agosto - diciembre 2019.

- Semestre septiembre 2020 - febrero 2021.

Durante los tres semestres mencionados a los alumnos se le impartió un taller de Scratch para aprender a usarlo, asimismo en 
ese tiempo desarrollaron pequeños juegos y animaciones básicas; este proceso sirvió de base al alumno para crear su videojuego ajustándose al aprendizaje basado en proyectos, el proceso fue llevado a cabo a la par del curso de Algoritmia. Los pasos seguidos se detallan a continuación:

Paso 1. Introducir al alumno al entorno de desarrollo del lenguaje de programación Scratch.

Paso 2. Desarrollar habilidadescompetencias para otorgar armas a los estudiantes para prepararse en la creación de sus propios videojuegos mediante pequeñas animaciones, programas y juegos básicos.

Paso 3. Formar equipos de trabajo colaborativo para el desarrollo de los videojuegos.

Paso 4. Emplear una estrategia metodológica, en este caso el aprendizaje basado en proyectos para diseñar, programar así como depurar sus videojuegos.

Paso 5. Presentar y evaluar los videojuegos.

Este proceso metodológico se ha venido aplicando durante las tres últimas ocasiones en donde se ha impartido Algoritmia y la muestra con la que se trabajó estuvo formada por un total de 66 alumnos quedando distribuidos como se detalla:

- Grupo 1 del semestre agosto diciembre 2018, 20 alumnos.

- Grupo 2 del semestre agosto diciembre 2019, 19 alumnos.

- Grupo 3 del semestre septiembre 2020 - febrero 2021, 27 alumnos.

Los datos de interés en esta investigación fueron las calificaciones finales de los videojuegos desarrollados las cuales fueron obtenidas mediante la evaluación de los trabajos de los diversos equipos de alumnos utilizando como instrumento de recolección una rúbrica para prácticas de programación (Vaca et al., 2014) cuyos ítems fueron contextualizados a la realidad investigativa mismos que estuvieron validados por personas expertas en el campo de ingeniería de software, de igual modo, este instrumento también incluyó las observaciones emitidas por el profesor a este respecto; en particular algunos aspectos de interés fueron los siguientes:

- Incluir las estructuras básicas de programación como son las estructuras de decisión, los ciclos, uso de operadores, variables, entre otros.

- Diseñar/documentar la narrativa del videojuego y recopilar toda información necesaria para crear el mismo.

- Crear/recopilar los componentes a emplear en el videojuego como los personajes, los fondos y los diversos sonidos.

- Programar el videojuego con interacción de dos usuarios de modo simultáneo.

- Incluir en el videojuego componentes de la cultura maya, lo cual es un factor primordial dado el contexto.

- Documentar el videojuego incluyendo el manual para el usuario.

El proceso se llevó a cabo durante 16 semanas, tiempo de duración de los semestres escolares de UADY, en función de esta situación, introducir a los alumnos a Scratch así como el desarrollo de habilidades y competencias para crear sus propios videojuegos tuvo una duración de 6 semanas, formar equipos de trabajo colaborativo se llevó a cabo en una semana y las 9 semanas restantes sirvieron para el diseño, programación, depuración, presentación así como también evaluación de los videojuegos.

\section{Resultados}

Como se ha mencionado, esta investigación se llevó a cabo en el lapso de tres semestres escolares, durante este tiempo han sido generados un total de 24 videojuegos, teniendo una misma cantidad de equipos colaborativos. Se incluye en este 
apartado una descripción breve de los proyectos más relevantes desarrollados (ver Tabla 1). Por otro lado, las Figura 1, Figura 2 y Figura 3 son un ejemplo de algunas de las imágenes diseñadas e incluidas en los diversos programas generados por los equipos de estudiantes.

\section{Tabla 1}

Videojuegos Finales de los Alumnos

\begin{tabular}{lll}
\hline Videojuego & Semestre & $\begin{array}{l}\text { Descripción } \\
\text { Quetzalcóatl debe moverse en pantalla y tratar de conseguir la } \\
\text { realización del solsticio de verano al llegar al final del camino. }\end{array}$ \\
\hline Ataque de los españoles & 1 & $\begin{array}{l}\text { Los españoles atacan a los mayas los cuales se defienden } \\
\text { esquivando las balas del cañón con ayuda de los dioses. }\end{array}$ \\
\hline Recolectando frutas & 1 & $\begin{array}{l}\text { Un soot's (murciélago personaje del pueblo maya, tiene el } \\
\text { encargo de atrapar frutas locales mientras le caen del cielo. }\end{array}$ \\
\hline Co'ox Kukulcan & 2 & $\begin{array}{l}\text { El dios maya Kukulcán deberá derrotar a un brujo el cual le robó } \\
\text { sus poderes. }\end{array}$ \\
\hline Siit' Canek & 2 & $\begin{array}{l}\text { Juego de tipo correr/saltar, en donde el jugador está escalando } \\
\text { una pirámide para ir acumulando puntos. }\end{array}$ \\
\hline El mundo maya & 2 & $\begin{array}{l}\text { Un estudiante se transporta a una época maya para derrotar a 4 } \\
\text { dioses: Chaac, Tlaltecuhtil, Ehecacózcatl y Kauil. }\end{array}$ \\
\hline Cabh's odissey & 2 & $\begin{array}{l}\text { El jugador se mueve en pantalla esquivando objetos los cuales } \\
\text { van cayendo desde el cielo. }\end{array}$ \\
\hline Conociendo: “Cultura maya” & 3 & $\begin{array}{l}\text { Juego donde se narra una historia relacionada con el pueblo } \\
\text { maya, considerando su arqueología, astronomía y cosmología. }\end{array}$ \\
\hline Oxlajuj & 3 & $\begin{array}{l}\text { Un maya astrólogo quiere compartir sus conocimientos, } \\
\text { predicciones, imaginaciones, etc. }\end{array}$ \\
\hline
\end{tabular}

Nota. 1 = semestre ago - dic 2018, 2 = semestre ago - dic 2019, 3 = semestre sep 2020 - feb 2021, elaboración propia (2021).

\section{Figura 1}

Videojuego: Aventura de Quetzalcóatl
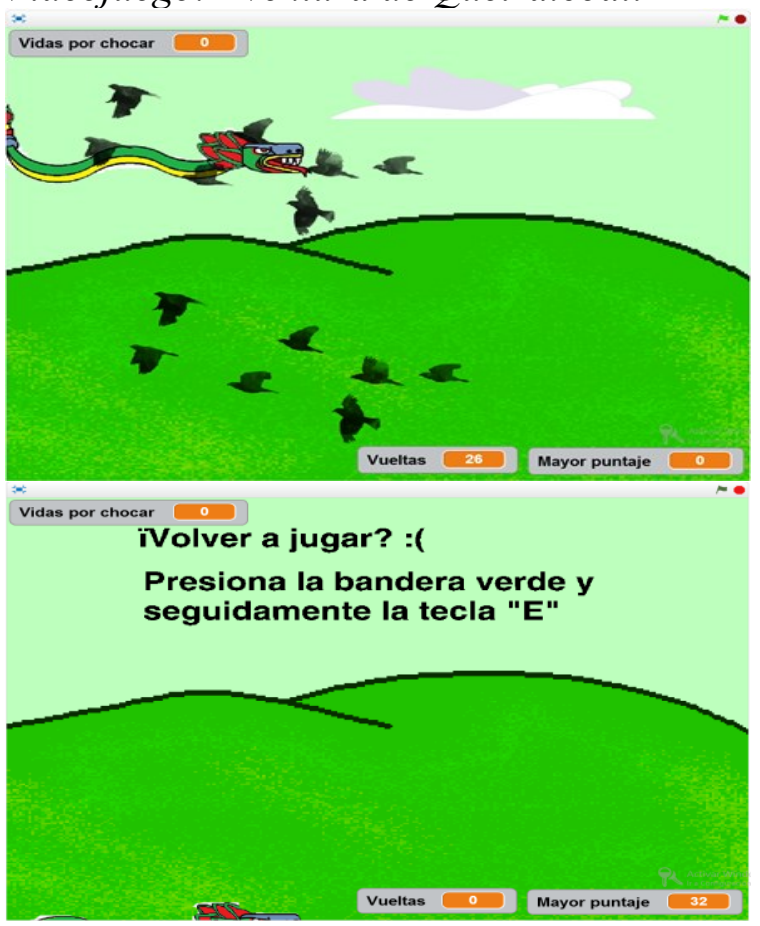

Nota. Se expone una imagen donde se observa a Quetzalcóatl viajando hacia su objetivo, así como también una pantalla para volver a iniciar una partida, elaborado por alumnos de Algoritmia (2018).
Figura 2

Videojuego: Cabh's odissey

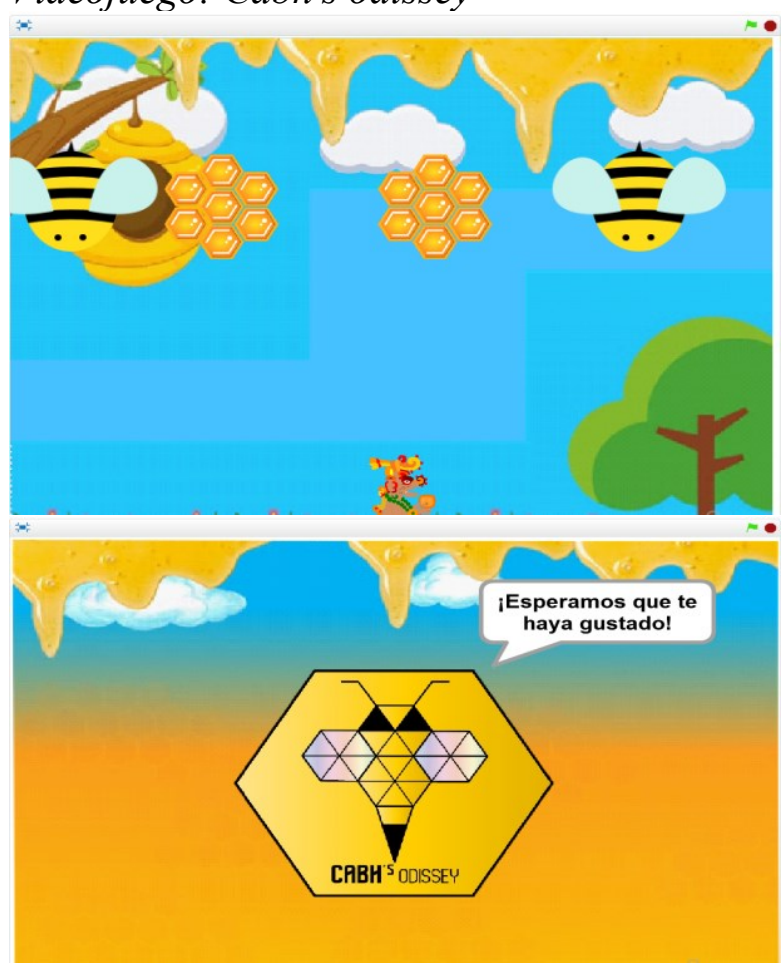

Nota. Se exhibe una imagen con el juego en acción, así como otra de despedida, elaborado por alumnos de Algoritmia (2019). 


\section{Figura 3}

\section{Videojuego: Oxlajuj}

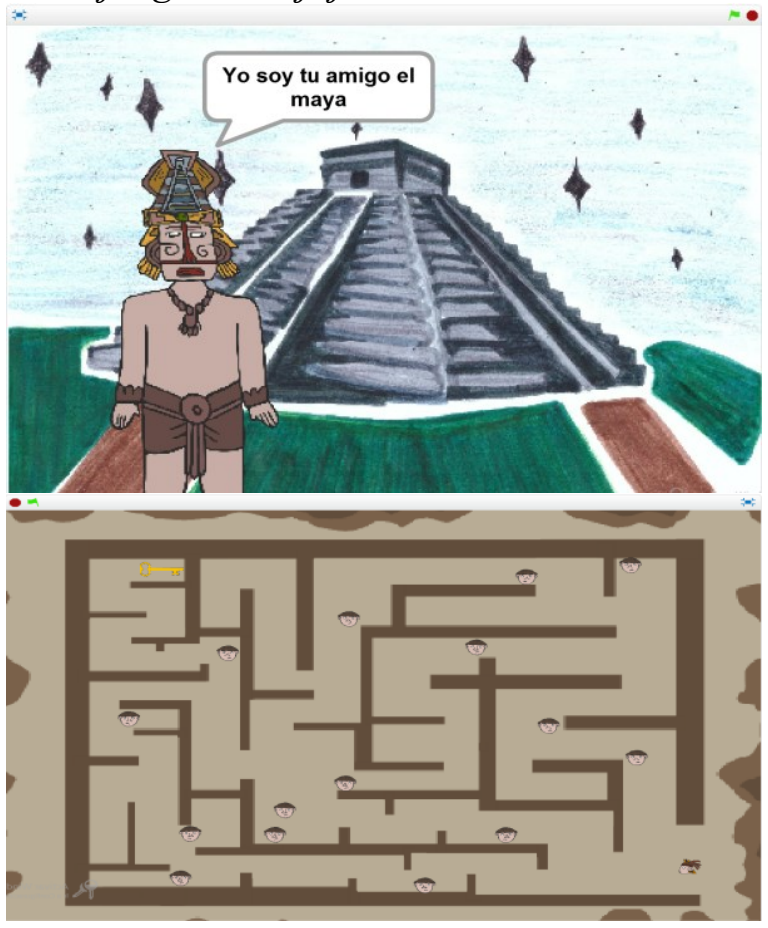

Nota. Se presenta una imagen con el personaje principal dando instrucciones y el laberinto a vencer, elaborado por Alumnos de Algoritmia (2021).

Por último, es este apartado, considerando el instrumento que fue utilizado para recabar los resultados finales que obtuvieron los alumnos en todo el proceso de creación de los videojuegos, se presenta en la Tabla 2 un condensado de las calificaciones de los participantes, haciendo especial mención al promedio logrado en cada uno de los tres periodos semestrales en los que se llevó a cabo la investigación, así como también el resultado global alcanzado durante este mismo tiempo.

\section{Tabla 2}

Resultados de los Videojuegos

\begin{tabular}{ll}
\hline Semestre & Promedio \\
\hline Agosto - Diciembre 2018 & 89.3 \\
\hline Agosto - Diciembre 2019 & 83.6 \\
\hline Septiembre 2020 & 86.3 \\
Febrero 2021 & \\
\hline Promedio general & $\mathbf{8 6 . 4}$
\end{tabular}

Nota. Detalle de las calificaciones finales obtenidas por los alumnos durante tres períodos semestrales, elaboración propia (2021).

\section{Discusiones}

En cuanto al promedio alcanzado como resultado de los videojuegos se observa lo siguiente: en cada uno de los tres semestres de interés este superaron los 80 puntos asimismo este resultado representa una calificación en el nivel de dominio satisfactorio de común acuerdo a los lineamientos de la UADY (2012), por otro lado, el promedio general de esos tres grupos fue de 86.4 puntos, el cual también se encuentra en el nivel satisfactorio lo que representa un valor adecuado para los efectos de esta investigación. Los resultados aquí obtenidos redundaron en un mejor aprovechamiento académico de los grupos de modo general.

Considerando la rúbrica de evaluación, se logró verificar en función de las observaciones emitidas por el profesor que todos los equipos de alumnos cumplieron con lo solicitado para el desarrollo de sus videojuegos, como fue la inclusión de las estructuras básicas de programación y en cuanto al diseño así como documentación de la narrativa del videojuego los equipos entregaron evidencia al respecto, todos los participantes consideraron aspectos relacionados con la cultura maya en los diversos personajes, fondos y sonidos, por último los equipos cumplieron entregando su documento final, así como también su manual de usuario.

Además de que todos los grupos incluyeron las estructuras básicas de programación, más del $70 \%$ de los mismos añadió estructuras las cuales estudiaron por cuenta propia como por ejemplo el uso de una cámara, realidad aumentada así como también la creación de módulos, lo anterior resultó relevante e inesperado, representando el claro interés de los alumnos en el desarrollo de sus trabajos. En cuanto al diseño así como documentación del videojuego, se realizó con los equipos sesiones personalizadas para aclarar puntos de mejora de lo presentado inicialmente, esta estrategia sirvió para obtener mejores productos finales. 
Es sustancial destacar el trabajo de los diversos equipos ya que incluyeron en sus proyectos no solo imágenes prediseñadas sino también aquellas creadas por ellos mismos; además inventaron sus propios personajes lo cual es un aspecto de suma importancia porque pudieron explorar su lado creativo a este respecto. En palabras del docente del curso, el $80 \%$ de los videojuegos entregados superó las expectativas porque no solo crearon su videojuego atendiendo las indicaciones iniciales, los diversos equipos se fueron un paso más adelante tanto en el diseño en general del videojuego como con las diversas opciones de interacción lo cual ha logrado marcar una diferencia. Como evidencia se pueden observar las Figura 4 y Figura 5 donde se muestran algunos de los objetos creados, así como también dos de los fondos empleados.

\section{Figura 4}

\section{Objetos Diseñados por los Alumnos}
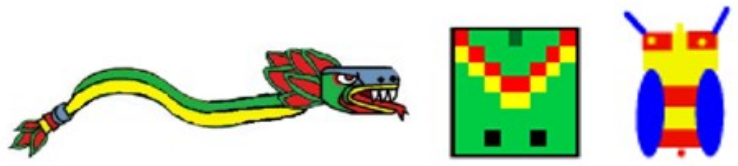

Nota. Objetos que se emplearon en los diversos videojuegos desarrollados durante tres períodos semestrales, elaborado por alumnos de Algoritmia (2019-2021).

\section{Figura 5}

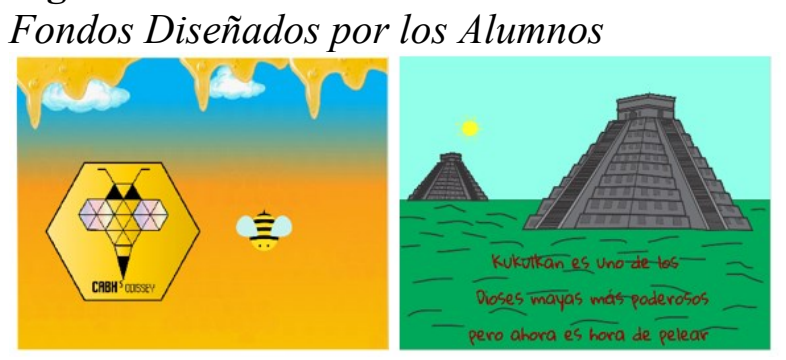

Nota. Se exponen dos de los fondos diseñados que se usaron en los videojuegos, elaborado por alumnos de Algoritmia (2019-2021).

\section{Conclusiones}

Adquirir los conceptos básicos de principios de programación suele presentarse como una tarea compleja para el alumno cuando recién inicia en cursos relacionados con esta área, sin embargo, el dominio en su uso es de vital importancia. Asignaturas como Algoritmia se vuelven el vehículo perfecto para desarrollar estrategias didácticas orientadas al área de programación de videojuegos mediante herramientas computacionales simples las cuales vayan acorde a los contenidos de los diversos cursos, donde el alumno pueda adquirir habilidades de pensamiento computacional para resolver problemas.

Partiendo de lo anterior se implementó un taller de Scratch resultando importante para apoyar Algoritmia porque trabaja apilando bloques con funciones predefinidas sin usar complejas sintaxis, además, es gratuito e intuitivo, útil para programar por ensayo y error; donde el alumno pudo desarrollar varios contenidos y competencias desde el razonamiento. Scratch posibilita el aprendizaje activo y constructivo donde los estudiantes son protagonistas de su proceso de aprendizaje lo cual los motiva. Scratch se emplea básicamente con niños de primaria y secundaria, sin embargo, tiene múltiples usos en otros niveles; Stuart (2009) plantea su empleo desde los primeros años de educación hasta introducir universitarios a la programación y concluye que es una buena alternativa para brincar posteriormente a otros lenguajes con entornos de desarrollo avanzados, lo cual es importante en el contexto.

El hecho de combinar el lenguaje de programación Scratch, la teoría del construccionismo y el aprendizaje basado en proyectos resultó de utilidad en Algoritmia, partiendo de esta mezcla se logró el desarrollo del pensamiento complejo en los estudiantes, dado que pudieron crear programas, animaciones, además de sus videojuegos con una herramienta tecnológica, de igual modo el alumno pudo aterrizar sus algoritmos en un programa tangible, entendiendo los conceptos más complejos.

El proceso metodológico empleado resultó adecuado en Algoritmia para resolver problemas de creatividad, análisis, planeación, entre otras, todas de importancia en el curso, se observó el compromiso además del interés de los educandos en el proceso, lo cual también se reflejó en los productos 
finales desarrollados. Los resultados de los proyectos fueron satisfactorios en función del nivel de dominio por medio del cual se rige la universidad, asimismo las observaciones del profesor se corresponden con este hecho.

Partiendo de integrar Scratch a Algoritmia mediante un taller, los objetivos de este artículo han sido cumplidos, esa integración sirvió de base para el alumno ya que pudo reforzar las estructuras estudiadas en el aula de clase construyendo un videojuego el cual reflejó el conocimiento adquirido; esta actividad representó el entendimiento por parte del alumno de los conceptos, dado que lo pudo explicar cabalmente a otro, en este caso a una computadora. Por último, es importante traer a colación el logro obtenido en el trabajo colaborativo ya que el desarrollo de los videojuegos fue en equipos en los cuales pudieron debatir sus ideas, llegando a consensos en todo el proceso de construcción.

$\mathrm{Se}$ vuelve relevante mencionar lo siguiente, partiendo del desarrollo de esta investigación también se lograron fomentar algunas competencias genéricas promovidas por la UADY en su Modelo Educativo de Formación Integral como son: comunicación en forma oral y escrita, el uso de tecnologías de información y comunicación, habilidades de investigación, el trabajo cooperativo, asimismo aplicación directa de sus conocimientos, como los aspectos más relevantes. Por otro lado, en este modelo se tiene una competencia sello relacionada con reconocer y valorar el entorno cultural, en este caso el maya, en consecuencia acorde a la investigación, también ha sido ampliamente fortalecida como se ha constatado en las descripciones de sus videojuegos.

En función de los resultados obtenidos en este proceso investigativo, los cuales han sido positivos hasta el momento, se espera seguir empleando el proceso metodológico aquí planteado en los semestres venideros del curso de Algoritmia en la Licenciatura en Ingeniería de Software, atendiendo al hecho de que se prevé se seguirán obteniendo buenos frutos y de este modo continuar ayudando a los alumnos a entender mejor los contenidos que se estudian en la materia.

\section{Reconocimiento}

Se agradece a todos los alumnos de Algoritmia de los semestres agosto diciembre 2018, agosto - diciembre 2019 así como también septiembre 2020 - febrero 2021, de la Licenciatura en Ingeniería de Software de la Unidad Multidisciplinaria Tizimín, UADY, los cuales formaron parte de este proceso investigativo, siendo los generadores de los videojuegos, objetos así como también de los fondos empleados en este artículo.

\section{Referencias}

Alania, R., Diez, C., \& Pinglo, J. (2012). Estrategias de enseñanza y estilos de aprendizaje en los alumnos del curso Introducción a la Algoritmia: caso Cibertec. [Tesis de Maestría]. Universidad Andrés Bello, Santiago de Chile, Chile.

Aparicio, O., \& Ostos, O. (2018). El Constructivismo y el Construccionismo. Revista Interamericana de Investigación, Educación y Pedagogía, RIIEP, 11(2), 115-120.

AulaPlaneta. (2021). Cómo crear videojuegos en tus clases. https://www.aulaplaneta.com/2021/01/11/recursos -tic/como-crear-videojuegos-en-tus-clases/

Compañ -Rosiqueet, P., Satorre-Cuerda, R., Llorens-Largo, F., \& Molina-Carmona, R. (2015). Enseñando a programar: un camino directo para desarrollar el pensamiento computacional. RED. Revista de Educación a Distancia (46). http://www.um.es/ead/red/46

Guerrero-Castaneda, R., Lenise do Prado, M., \& OjedaVargas, M. (2016). Reflexión crítica epistemológica sobre métodos mixtos en investigación de enfermería. Enfermería Universitaria, 13(4). https://www.sciencedirect.com/science/article/pii/ S1665706316300422

Gómez, J., Narváez, L., Rejón, E., \& Reyes, J. (2016). Planeación didáctica de Algoritmia. Universidad Autónoma de Yucatán, Mérida, México.

González, J., Cabrera, M., \& Gutiérrez, F. (2007). Diseño de videojuegos aplicados a la Educación Especial. Actas del VIII Congreso Internacional de Interacción Persona Ordenador (INTERACCIÓN 2007), (35-44).

López, J. (2011). Programación con Scratch. Eduteka. http://eduteka.icesi.edu.co/pdfdir/AlgoritmosProgr amacionCuaderno1.pdf

López-Escribano, C., \& Sánchez-Montoya, R. (2012). Scratch y Necesidades Educativas Especiales: Programación para todos. RED. Revista de 
Educación a Distancia. https://www.redalyc.org/html/547/54724753001/

Monjelat, N., Cenacchi, M., \& San Martín, P. (2018). ¿Programación para Todos? Herramientas y Accesibilidad: Un Estudio de Caso. Revista Latinoamericana de Educación Inclusiva, 12(1), 213-227.

http://www.rinace.net/rlei/numeros/vol12num1/art12.pdf

Muñoz, R., Barcelos, T., Villarroel, R., \& Silveira, I. (2015). Diseño e implementación de un taller de programación de juegos digitales con Scratch como apoyo a Fundamentos de Programación. Anais dos Workshops do IV Congresso Brasileiro de Informática na Educacao (CBIE), (1495-1504). http://dx.doi.org/10.5753/cbie.wcbie.2015.1495

Narváez, L., \& López, R. (2019). Actitudes de los alumnos de la asignatura de algoritmia ante el uso del lenguaje de programación Scratch. Congreso Internacional de Investigación Academia Journals, (2492-2497). Celaya.

Saavedra, E. (2020). Beneficios educativos de la creación de juegos y videojuegos. Vermislab. https://www.vermislab.com/game-makersbeneficios-educativos-de-la-creacion-de-juegos-yvideojuegos/

Stuart, G. (2009). Learning to program from Scratch. 9th IEEE International Conference on Advanced Learning Technologies, ICALT 2009, (451-452). Riga, Latvia. doi:10.1109/ICALT.2009.50

Team, MIT Scratch. (2018). Scratch. http://scratch.mit.edu/

Universidad Autónoma de Yucatán (UADY). (2012). Modelo educativo para la formación integral. Universidad Autónoma de Yucatán, Mérida, México.

Vaca, J., Agudo, J., \& Sánchez, H. (2014). Evaluación de prácticas de programación mediante rúbricas en Moodle. Actas de las XX JENUI. Oviedo. https://upcommons.upc.edu/bitstream/handle/2099 /15458/P107va_eval.pdf

Vargas, C. (2015). La creación de videojuegos en ciencias naturales y la competencia para resolver problemas. Revista Lasallista de Investigación, 12(2). 